\title{
Novo método para seleção de dispersantes de última geração para concretos refratários
}

\section{(Novel method for the selection of enhanced dispersants for refractory castables)}

\author{
A. R. F. Pardo, R. G. Pileggi, V. C. Pandolfelli \\ Universidade Federal de S. Carlos, DEMa \\ Rod. Washington Luiz, km 235, C.P. 676, 13565-905, S. Carlos, SP \\ parfp@iris.ufscar.brouvicpando@power.ufscar.br
}

\begin{abstract}
Resumo
O aprimoramento dos processos de instalação de concretos refratários (auto-escoamento, bombeamento, projeção, etc.) tem intensificado a necessidade de otimização e controle do comportamento reológico destes materiais. A distribuição granulométrica e a concentração de sólidos, além da natureza das matérias-primas e dos aditivos químicos presentes no sistema, são os principais fatores que determinam sua reologia. Em conseqüência, a correta escolha dos agentes dispersantes é uma das tarefas fundamentais para o desenvolvimento sistemático de concretos. Contudo, os critérios tradicionalmente empregados para esta seleção, os quais se baseiam no ensaio de fluidez ou na caracterização reológica da matriz, não avaliam o comportamento do material frente às solicitações diversas de seu processamento e de sua aplicação. Sendo assim, neste trabalho foi proposto um novo método para avaliação e seleção de dispersantes, o qual analisa o comportamento do concreto desde a etapa de mistura até seu fluxo sob cisalhamento intenso. Dois aditivos usualmente empregados em concretos de alta alumina, polimetacrilato de sódio e ácido cítrico, além de dois novos dispersantes de última geração, foram então avaliados pelo método proposto e pela tradicional fluidez. Os resultados demonstraram a eficiência da nova técnica, além de atestarem o superior desempenho dos novos dispersantes avaliados.
\end{abstract}

Palavras-chave: concreto, aditivos, reômetro, refratários, dispersantes.

\begin{abstract}
The continuous evolution in the installation techniques of refractory castables (self-flowing, pumpable, shotcrete, etc.) has intensified the requirements for optimization and control of the rheological behavior of such materials. The main factors that control the rheology of castables are the particle size distribution, the solid concentration, and the nature of the raw-materials and chemical additives present in the system. Therefore, the right choice of dispersing agents is very important for the systematic development of these materials. However, the selection is traditionally based on castables' free-flow or matrix rheology measurements, which do not evaluate the material's behavior during the distinct steps of its production and application. In this study, a novel method based on the rheometry of castables is proposed for the evaluation and selection of dispersants. Unlike usual techniques, the present one allows the evaluation of the rheological behavior from the mixing step until the flow under intensive shearing. Additives usually applied in high alumina castables, sodium polymethacrylate and citric acid, and two dispersants based on a new molecular design were evaluated by the proposed method and by the traditional free-flow technique. Results demonstrated the advantages of the proposed characterization method and demonstrated the superiority of the novel additives.
\end{abstract}

Keywords: castables, refractories, rheology, dispersants, rheometry.

\section{INTRODUÇÃO}

A característica marcante dos concretos refratários está em seu processamento e sua aplicação serem realizados no estado fluido. Ao longo dos anos, diversas técnicas têm sido desenvolvidas com intuito de aumentar a eficiência e reduzir o tempo e os custos de moldagem. Os esforços atuais concentramse na evolução das composições refratárias em sintonia com o aprimoramento dos processos passíveis de automação: bombeamento e projeção.

Esta tendência tem motivado diversos estudos [1-10] envolvendo os fatores que influenciam o comportamento reológico destes materiais, como a distribuição granulométrica, a concentração de sólidos, a natureza das matérias-primas e a atuação de aditivos químicos (dispersantes, surfactantes, modificadores de viscosidade, etc.).

Tradicionalmente, estes estudos são baseados no clássico ensaio de fluidez [1] ou na caracterização reológica da matriz (partículas $<100 \mu \mathrm{m})$ [2]. Há, portanto, uma defasagem entre as crescentes exigências tecnológicas dos concretos e os limitados procedimentos de análise.

Após o surgimento de um reômetro específico para 
concretos refratários [3], esta lacuna tem sido preenchida pela substituição dos procedimentos de ensaios convencionais por outros que utilizam a reometria propriamente dita.

Utilizando-se a técnica de reometria, este trabalho teve como objetivo desenvolver um novo método para avaliação da influência que os aditivos dispersantes exercem sobre o comportamento reológico dos concretos refratários em todas as etapas de seu processamento: mistura, transporte, moldagem e endurecimento.

O método proposto foi utilizado na avaliação de dois dispersantes com arquitetura molecular linear amplamente utilizados em concretos de alta alumina (polimetacrilato de sódio e ácido cítrico), além de outros dois considerados de última geração, cujas moléculas são do tipo pente. Os aditivos foram testados nos sistemas alumina - cimento e alumina - cimento - microssílica, sendo os resultados obtidos comparados ao ensaio tradicional de fluidez.

\section{MATERIAISE MÉTODOS}

Duas composições de concretos auto-escoantes de alta alumina ( $0 \%$ e $4 \%$ em volume de microssílica) e ultra-baixo teor de cimento foram formuladas segundo o modelo de distribuição granulométrica de Andreasen, com coeficiente $\mathrm{q}=0,21$ e diâmetro máximo de $4750 \mu \mathrm{m}$. O teor de água utilizado foi de $15 \%$ em volume.

A Tabela I apresenta as duas composições elaboradas. Os dispersantes avaliados neste trabalho encontram-se listados na Tabela II, sendo descritos os teores empregados, o peso molecular e a estrutura da moléculas.

Tabela I - Composições dos concretos de alta alumina (\% em peso).

[Table I - High alumina castables' compositions (wt. \%).]

\begin{tabular}{lccc}
\hline Materias-primas & $\begin{array}{c}\text { alumina- } \\
\text { cimento } \\
\% \text { peso }\end{array}$ & $\begin{array}{r}\text { alumina } \\
\text { cimento } \\
\text { microssílica } \\
(\% \text { peso })\end{array}$ \\
\hline & $\begin{array}{c}\text { Microssílica } \\
\text { Aluminas }\end{array}$ & 0,0 & 2,1 \\
& Calcinadas & 21,6 & 19,1 \\
$\begin{array}{l}\text { Matriz } \\
(<100 \mu \mathrm{m})\end{array}$ & $\begin{array}{c}\text { ALO Marrom 200/F } \\
\text { Cimento CA 14 }\end{array}$ & 25,3 & 21,6 \\
\hline $\begin{array}{l}\text { Agregado } \\
(<100 \mu \mathrm{m})\end{array}$ & ALO Branco & 51,6 & 1,7 \\
\hline
\end{tabular}

Obs: Aluminas calcinadas $=$ A1000 SG e A3000 FL; CA $14=$ cimento de aluminato de cálcio $(\mathrm{CAC}) ; \mathrm{ALO}$ Branco = alumina eletrofundida branca. Todas as matérias-primas foram fornecidas pela empresa Alcoa Alumínio S.A. As composições foram formuladas através do software PSDesigner [1].

[Note: calcined alumina $=$ A1000 SG and A3000 FL; CA $14=$ calcium aluminate cement; $W F A=$ white fused alumina. The raw materials were supplied by Alcoa-Brazil and US. The formulation was calculated using the PSDesigner [1] software program.]
Tabela II - Dispersantes avaliados, teores empregados e algumas de suas características: peso molecular e arquitetura de cadeia. [Table II - List of dispersants analyzed in this work, their applied contents, and some of their characteristics: molecular weight and molecule design.]

\begin{tabular}{lccc}
\hline Aditivo & Teor & Peso Molecular & Arquitetura \\
& $\mathrm{mg} / \mathrm{m}^{2}$ & $\mathrm{~g} / \mathrm{mol}$ & Molecular \\
\hline
\end{tabular}

Polimetacrilato

\begin{tabular}{lccc} 
de Sódio $(\mathrm{DV})^{*}$ & 0,40 & 2500 & Linear \\
\hline Ácido cítrico $(\mathrm{AC}) * *$ & 0,26 & 192 & Linear
\end{tabular}

Policarboxilato

\begin{tabular}{llll}
\hline Éter $(\mathrm{FS} 10)^{* * *}$ & 0,78 & 10000 & Pente \\
Policarboxilato & & \\
\hline
\end{tabular}

\begin{tabular}{llll}
\hline Éter $(\mathrm{FS} 20)^{* * *}$ & 0,78 & 8000 & Pente \\
\hline
\end{tabular}

*Darvan 7S, Vanderbilt, EUA; ** Synth e *** SKW Polymers Gmbh, Alemanha. O aditivo FS10 é um derivado do polietilenoglicol, sendo indicado para concretos de alta alumina isentos de microssílica. O FS20, que é baseado em um policarboxilato, foi desenvolvido para sistemas contendo $\mathrm{SiO}_{2}$.

[* Darvan 7S, Vanderbilt, EUA; ** Synth and *** SKW Polymers Gmbh, Alemanha. FS10 is derived from polyethyleneglycol, and is indicated for silica-free castables. FS20, is based on polycarboxylate, and is suitable to $\mathrm{SiO}_{2}$ containing systems.]

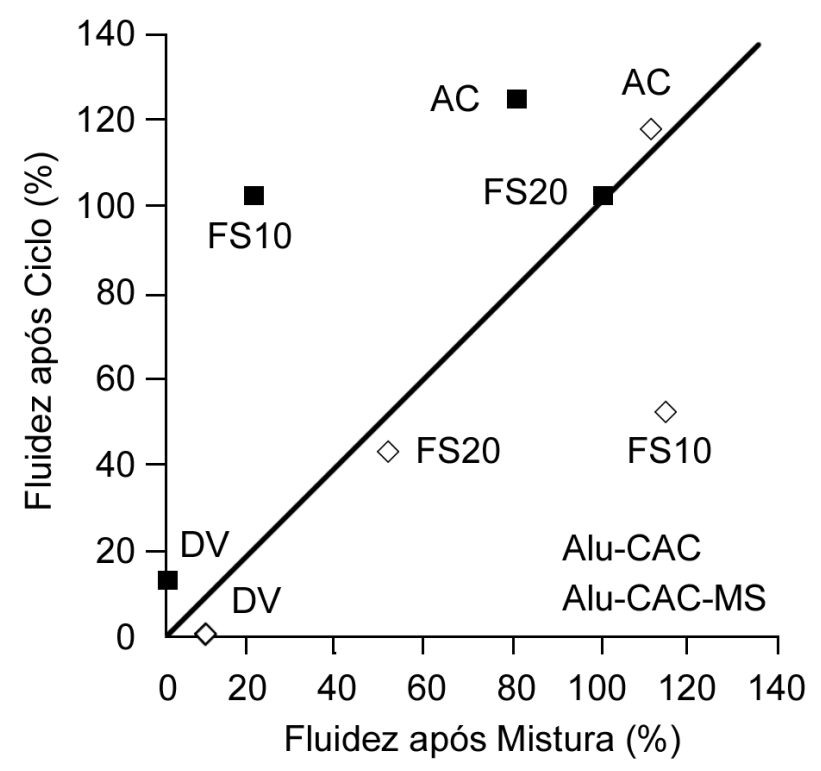

Figura 1: Valores de fluidez livre (\%) medidos após a mistura e ciclo de cisalhamento, das composições alumina - cimento (Alu-CAC) e alumina - cimento - microssílica (Alu-CAC-MS), processadas com os quatro aditivos testados. Obs: região indicada para os concretos auto-escoantes $(80 \%<\mathrm{FL}<110 \%)$ está destacada em cinza. A reta na diagonal indica valores iguais de fluidez após mistura e ciclo.

[Figure 1: Influence of the tested additives on the alumina-cement (Alu$C A C)$ and alumina-cement-microsilica (Alu-CAC-MS) free-flow values (\%) after mixing and after the shearing cycle. Note: the flow range for self-flowing castables is delimited by the gray area. The diagonal line indicates similar flow values after mixing and shearing cycles.] 
Todas as composições foram misturadas e ensaiadas no reômetro para concretos segundo a seqüência experimental proposta pelo novo método de avaliação dos dispersantes, a qual é descrita a seguir:

\section{a) Etapa 1: Avaliação da mistura, fluidez e natureza reológica}

1. Mistura sob rotação constante de $33 \mathrm{rpm}$, sendo a água adicionada de modo continuo à taxa de $8 \mathrm{~g} / \mathrm{s}$. Após a virada, os concretos foram adicionalmente homogeneizados por mais 2 minutos;

2. Medida da fluidez livre (FL) imediatamente após a mistura, de acordo com a norma ASTM C 860 adaptada para concretos auto-escoantes;

3. Caracterização reológica através de um ciclo de cisalhamento (entre 2 rpm e $75 \mathrm{rpm}$ ) sem restrição de volume, seguida de uma nova determinação da fluidez.

\section{b) Etapa 2: Avaliação do endurecimento do concreto (trabalhabilidade)}

Trabalhabilidade, pode ser definida como o tempo necessário para que a fluidez livre atinja valores inferiores a $30 \%$. Sua medida foi realizada de duas distintas maneiras

1. Trabalhabilidade estática: após a mistura e determinação inicial da fluidez (procedimentos anteriormente descritos), os concretos foram armazenados em um recipiente hermeticamente fechado para inibir a evaporação da água. A fluidez foi sucessivamente avaliada, respeitando-se o intervalo de 15 minutos entre cada medida;

2. Trabalhabilidade dinâmica: após a mistura, as composições foram mantidas no reômetro sob cisalhamento contínuo à rotação constante de $28 \mathrm{rpm}$. A cada 15 minutos, a rotação foi reduzida a zero para que também fosse medida a variação da fluidez livre.

\section{RESULTADOS E DISCUSSÃO}

A abordagem tradicional de caracterização reológica de concretos baseada no ensaio de fluidez livre, é o ponto inicial para a análise da influência exercida por distintos dispersantes (DV, AC, FS 10 e FS 20) na reologia dos sistemas alumina - cimento (Alu-CAC) e alumina - cimento - microssílica (Alu-CAC-MS).

A Fig. 1 compara os resultados de fluidez livre (FL) das composições medidos após a mistura com os valores obtidos depois do ciclo de cisalhamento. Como observado, com exceção do DV, para alguma composição ou condição em que a medida foi efetivada, os aditivos proporcionaram uma adequada dispersão aos concretos, atingido níveis de fluidez na região de auto-escoabilidade $(80 \%<\mathrm{FL}<110 \%)$.

Pode-se afirmar que o AC dispersou adequadamente ambos os sistemas estudados (FL $>80 \%$ ), enquanto o FS 10 foi mais eficiente no Alu-CAC, necessitando de uma energia de cisalhamento extra, fornecida pelo ciclo, para dispersar o sistema Alu-CAC-MS. O FS 20, por sua vez, foi efetivo apenas no sistema Alu-CAC-MS, confirmando a indicação de seu fabricante [4].

Como observado, os resultados de fluidez não apresentaram

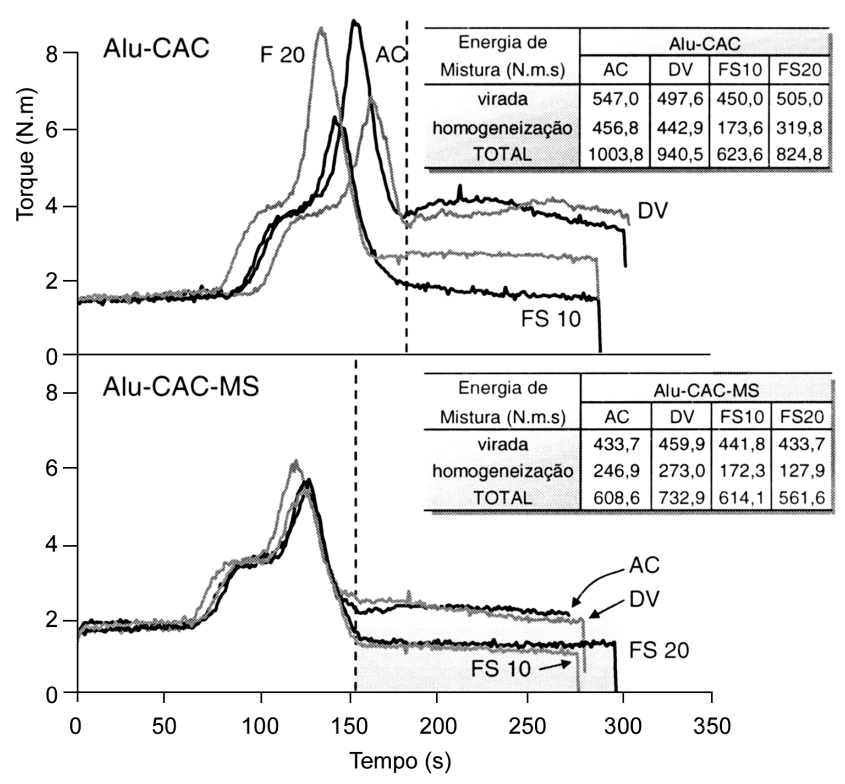

Figura 2: Comportamento de mistura das composições alumina cimento (Alu-CAC) e alumina - cimento - microssílica (Alu-CACMS), processadas com os quatro aditivos testados. As tabelas apresentam os valores estimados de energia de mistura (área sob a curva torque $\mathrm{x}$ tempo) para a etapa da virada, para a homogeneização e sua soma total. Obs: a etapa de mistura / virada dos concretos está delimitada pela linha pontilhada. O período de homogeneização é destacado pela região em cinza.

[Figure 2: Influence of the tested additives on the alumina-cement (Alu$C A C)$ and alumina-cement-microsilica (Alu-CAC-MS) mixing behavior. The attached tables display the estimated mixing energy values (area under the curve of torque $x$ time), indicating the energy consumed in the turning point region and in the homogenization step. Note: the turning point region is delimited by the dotted line and the homogenization step is enclosed in the gray area.]

nenhuma vantagem para os novos aditivos, FS 10 e FS 20, em relação ao AC. Contudo, este tipo de ensaio avalia somente a característica de moldagem do material, sendo incapaz de fornecer informações sobre sua verdadeira natureza reológica e seu desempenho nas demais fases do processamento dos concretos: mistura, transporte e endurecimento em função do tempo (trabalhabilidade).

Uma provável solução para este problema é a integração das técnicas de reometria de concretos e fluidez, como proposto neste trabalho. Os resultados obtidos por este novo método são discutidos a seguir.

\section{a) Etapa 1: Mistura, Fluidez e Natureza Reológica}

As composições (Alu-CAC e ALU-CAC-MS) foram misturadas no reômetro à rotação constante de $33 \mathrm{rpm}$, sendo a água adicionada continuamente à taxa de $8 \mathrm{~g} / \mathrm{s}$. A análise dos resultados (Fig. 2) requer uma prévia discussão sobre os fenômenos envolvidos neste processo.

De uma maneira geral, a adição de água em um sistema de partículas leva à formação de aglomerados devido às forças de van der Waals e à atuação das forças capilares [5]. Para reduzir a aglomeração, dois mecanismos distintos são utilizados: (a) cisalhamento mecânico e (b) ação dos dispersantes. 


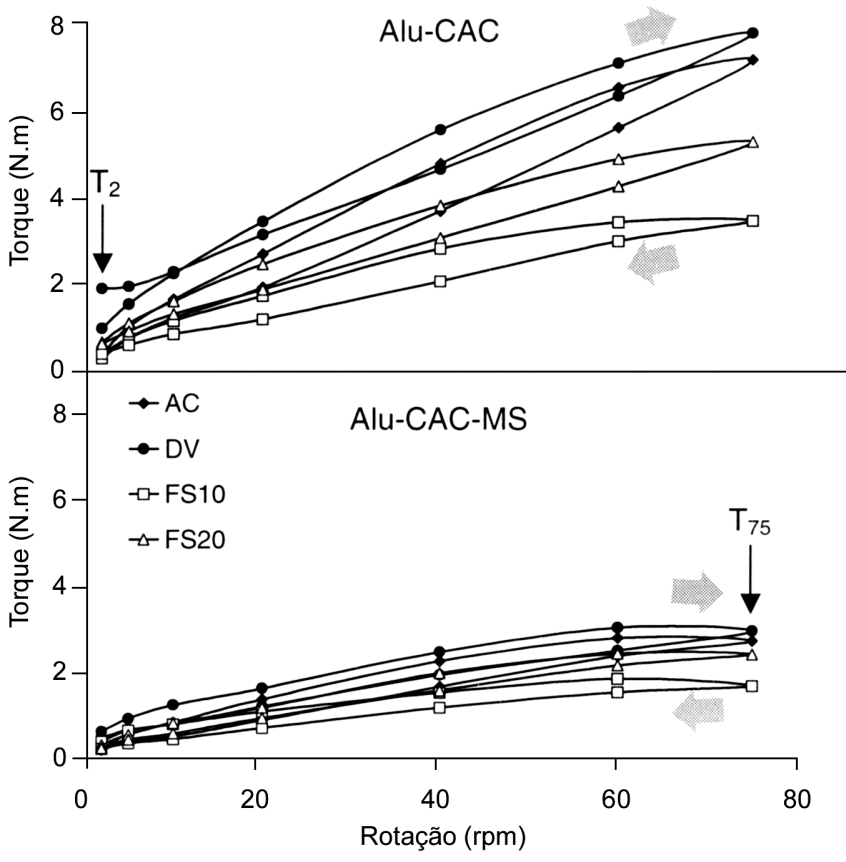

Figura 3: Ciclos de cisalhamento (entre 2 e $75 \mathrm{rpm}$ ) obtidos no reômetro para concretos refratários [3] das composições alumina - cimento (AluCAC) e alumina - cimento - microssílica (Alu-CAC-MS), processadas com os quatro aditivos testados. Obs: setas em cinza indicam o sentido de aplicação da rotação.

[Figure 3: Refractory castables shearing cycles (between 2 rpm and 75 rpm) for the alumina-cement (Alu-CAC) and alumina-cement-microsilica (Alu-CAC-MS) compositions prepared with the four distinct tested additives. Note: the speed variation is shown by the gray arrows.]

O instante de virada do concreto ocorre quando os sistemas atingem uma certa distribuição de unidades móveis (partículas e/ ou aglomerado), a qual leva ao surgimento de pontes líquidas de ligação [5], provocando um drástico aumento na resistência ao cisalhamento. Quanto maior a área superficial destas unidades, mais pontes são formadas, elevando os esforços necessários para mistura da massa, além de intensificar o atrito entre as partículas.

A manutenção do cisalhamento em sincronia com a atuação dos dispersantes, rompe esta estrutura, causando o desaparecimento das pontes (água é liberada para aumentar a distância entra as partículas) e a instantânea redução da resistência ao cisalhamento (diminuição do torque). A partir deste momento, os concretos passam a manifestar sua natureza reológica, comportando-se como fluidos.

A Fig. 2 apresenta as curvas de mistura obtidas com os quatro dispersantes testados. Como um mesmo procedimento de mistura foi adotado para todas as composições, os aditivos foram os responsáveis pelos comportamentos observados.

No sistema Alu-CAC, os dispersantes exerceram profunda influência na mistura, tanto na região de virada, como na homogeneização, conforme discutido a seguir:

1. Ácido Cítrico (AC): a composição contendo $\mathrm{AC}$ apresentou elevado torque na virada $(8,7$ N.m) e utilizou uma grande parcela da energia total para a etapa de mistura (estimada pela área sob a curva torque $\mathrm{x}$ tempo), como apresentado na tabela inserida na Fig. 2.
Surge assim um aparente paradoxo: como o aditivo que proporcionou a maior fluidez livre ( $\mathrm{FL}=112 \%$, Fig. 1) consome maior energia para virada e homogeneização?

A alta fluidez é decorrente da eficiente dispersão produzida por este aditivo, a qual resulta na individualização das partículas e por conseqüência na elevação da área superficial das unidades móveis no sistema, dificultando a mistura do material. Este comportamento é ainda mais acentuado pela reduzida espessura da molécula de $\mathrm{AC}(\approx 0,5 \mathrm{~nm})$, a qual permite uma maior aproximação entre as partículas, intensificando os fenômenos de atrito entre as mesmas. Desta maneira, o esforço necessário para mistura aumenta significativamente, tanto na virada, como no período de homogeneização.

2. Polimetacrilato de Sódio (DV): o reduzido valor de torque na virada (6,7 N.m) obtido pela composição com DV resulta da inadequada dispersão produzida por este aditivo, como visto na Fig. $1(\mathrm{FL}=9 \%)$. Neste caso, as unidades móveis são aglomerados de partículas com reduzida área superficial. Contudo, a maior dificuldade de escoamento deste sistema aumenta a energia necessária para sua mistura.

No caso do DV, o alto peso molecular $(2500 \mathrm{~g} / \mathrm{mol})$ é distribuído em uma molécula com arquitetura linear, resultando em cadeias longas ( $\approx 8 \mathrm{~nm}$ a $9 \mathrm{~nm}$ ). Isto aumenta a probabilidade da ocorrência dos fenômenos de "bridging" e "depletion" $[6,7]$ entre as partículas, dificultando a dispersão dos concretos em concentrações de sólidos elevadas, como a utilizada neste trabalho (85\% em volume de sólidos).

3. Policarboxilato Éter (FS 10): o aditivo FS 10 resultou nos menores valores de torque na virada (6,3 N.m) e de energia de mistura, apesar de também ter propiciado uma adequada dispersão ao concreto $(\mathrm{FL}=115 \%)$. Isto indica que os fenômenos ativados pela individualização das partículas durante a mistura foram de alguma forma compensados pela molécula do FS 10 .

Seu elevado peso molecular $(10000 \mathrm{~g} / \mathrm{mol})$, distribuído na arquitetura de um pente, resulta em uma molécula com reduzidas dimensões $\left(\approx 3,0 \times 3,2 \mathrm{~nm}^{2}\right.$ - valores fornecidos pelo fabricante), com elevada densidade de cargas (negativa) na cadeia principal, mas com uma camada estérica com características lubrificantes, originadas pelas cadeias laterais [4, 8-10]. O FS 10 unifica, portanto, as vantagens dos polieletrólitos de elevado peso molecular, com a facilidade de movimentação dos aditivos de cadeia curta.

4. Policarboxilato Éter (FS 20): o aditivo FS 20 possui um elevado peso molecular $(8000 \mathrm{~g} / \mathrm{mol})$, mas sua geometria molecular de sua cadeia tipo pente $\left(\approx 0,5 \times 24 \mathrm{~nm}^{2}\right.$-valores fornecidos pelo fabricante), resulta em uma camada estérica com dimensões próximas ou levemente superiores $(>0,5 \mathrm{~nm})$ que a do $\mathrm{AC}$. Como conseqüência, a composição dispersada com este aditivo obteve torque na virada $(8,6 \mathrm{~N} . \mathrm{m})$ similar ao do AC.

Apesar de não ter resultado na máxima dispersão das partículas $(\mathrm{FL}=51,3 \%)$, como esperado para sistemas sem microssílica, os níveis de energia de mistura na etapa de virada e de homogeneização, mostraram-se bastante reduzidos, provavelmente devido às características lubrificantes de suas cadeias laterais e não à individualização das unidades móveis.

A introdução de microssílica no sistema Alu-CAC-MS, modificou as curvas de mistura de todas as composições, reduzindo e aproximando os valores de torque máximo e de energia de mistura 


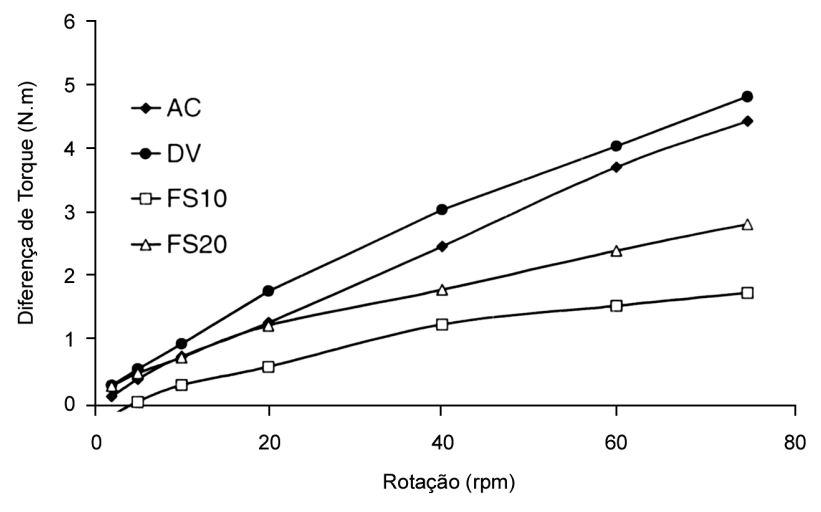

Figura 4: Diferença calculada entre os níveis de torque registrados pelas composições alumina - cimento (Alu-CAC) e alumina - cimento - microssílica (Alu-CAC-MS), processadas com os quatro aditivos testados.

[Figure 4: Calculated torque differences between the alumina-cement (Alu-CAC) and alumina-cement-microsilica (Alu-CAC-MS) compositions prepared with the four distinct tested additives.]

para os quatro aditivos testados (Fig. 2).

Este resultado está associado com o reduzido diâmetro de suas partículas esféricas ultra-finas $(\approx 0,1 \mu \mathrm{m})$. Estas características geométricas permitem sua atuação como rolamentos, facilitando a movimentação relativa das maiores partículas, e assim reduzindo os fenômenos de atrito no sistema.

Além da contribuição física, a microssílica geralmente reduz o $\mathrm{pH}[7,11]$ do concreto, modificando a natureza das interações entre os aditivos e a superfície das partículas. Por esta razão, os aditivos atuaram de maneira distinta nas composições estudadas, justificando a maior eficiência do FS 20 no sistema Alu-CAC-MS.

A fluidez livre indica o estado de dispersão de uma composição, apresentando uma relação inversa com sua tensão de escoamento (tensão mínima para início do escoamento) [7]. Está portanto relacionada com as características de fluxo do material em rotações próximas a zero. Uma vez que durante a homogeneização as composições foram mantidas a $33 \mathrm{rpm}$, o que se observou neste período foi a manifestação das características viscosas da reologia dos concretos. A investigação detalhada da natureza reológica das composições foi realizada através dos ciclos de cisalhamento (Fig. 3).

Os valores de torque a $2 \mathrm{rpm}\left(\mathrm{T}_{2}\right)$, os quais refletem a tensão de escoamento, apresentaram relação inversa com a fluidez das composições. Contudo, os aditivos exerceram influência realmente significativa no comportamento dos concretos em elevadas rotações.

No sistema Alu-CAC as composições apresentaram-se pseudoplásticas, com grande diferenciação entre os valores de torque a $75 \mathrm{rpm}\left(\mathrm{T}_{75}\right)$. Os aditivos de cadeia linear, $\mathrm{AC} \mathrm{eDV}$, resultaram em elevados níveis de $\mathrm{T}_{75}$, ao contrário daqueles do tipo pente, $\mathrm{FS}$ 10 e FS 20. As características lubrificantes destes novos aditivos, principalmente do FS 10, facilitam o deslizamento relativo entre as partículas em condições severas de cisalhamento, fato que pode ser útil em aplicações como o bombeamento e a projeção.

A inclusão da microssílica no sistema Alu-CAC-MS resultou no comportamento pseudoplástico, com reduzidos valores de $\mathrm{T}_{75}$ para todos os concretos. Assim como na mistura, as partículas esféricas da microssílica facilitaram a movimentação, reduzindo o atrito no sistema.

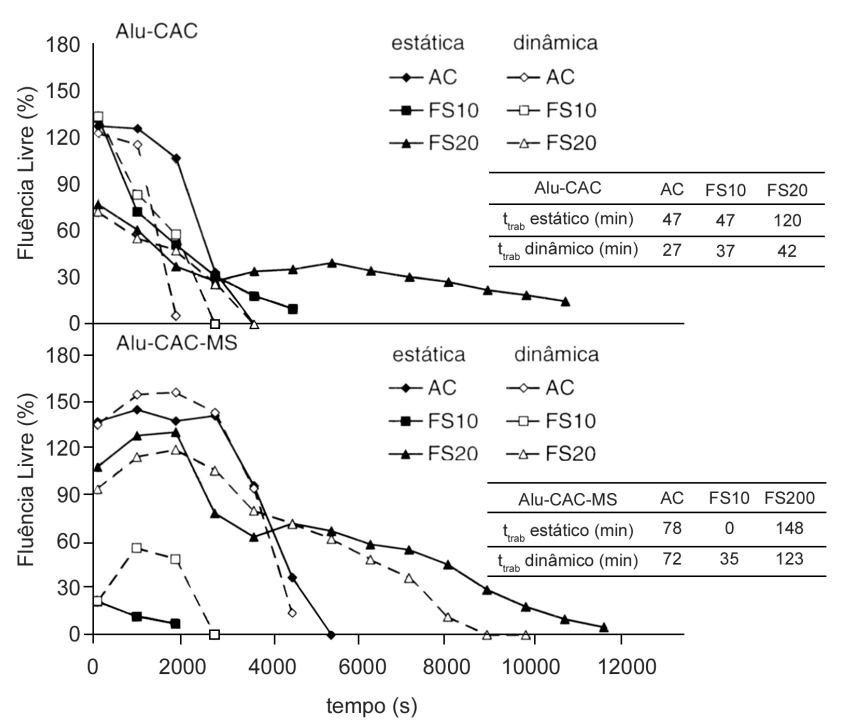

Figura 5: Variação da fluidez em função do tempo (trabalhabilidade), medida através de ensaios estáticos e dinâmicos, para as composições alumina cimento (Alu-CAC) e alumina - cimento - microssílica (Alu-CAC-MS), processadas com ácido cítrico, FS 10 e FS 20. Obs: faixa em cinza referese à fluidez abaixo de $30 \%$; faixa destacada por riscos diagonais em cinza delimita a região de auto-escoamento $(80 \% \leq \mathrm{FL} \leq 100 \%)$; tabelas apresentam o tempo de trabalhabilidade $\left(\mathrm{t}_{\text {trab }}\right)$ expresso em minutos.

[Figure 5: Static and dynamic evaluation of castables hardening (freeflow value along the time - workability) for the alumina-cement (Alu$C A C)$ and alumina-cement-microsilica (Alu-CAC-MS) compositions prepared with citric acid, FS 10 and FS 20. Note: flow values lower than $30 \%$ are enclosed in the gray area; the flow range for self-flowing castables is delimited by the dashed area; the attached tables display the workability time $\left(t_{\text {trab }}\right)$.]
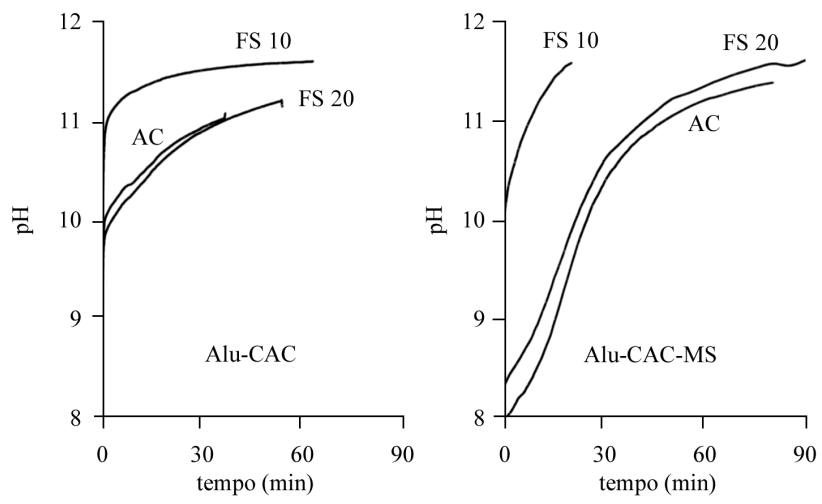

Figura 6: Variação de $\mathrm{pH}$ em função do tempo para as composições alumina - cimento (Alu-CAC) e alumina - cimento - microssílica (AluCAC-MS), processadas com ácido cítrico, FS 10 e FS 20.

[Figure 6: $p H$ changes along time for the alumina-cement (Alu-CAC) and alumina-cement-microsilica (Alu-CAC-MS) compositions prepared with citric acid, FS 10 and FS 20.]

Através da subtração dos valores de torque entre os sistemas Alu-CAC e Alu-CAC-MS durante o ciclo de cisalhamento (Fig. 4), observa-se que os aditivos com arquitetura pente, principalmente o FS 10 , tornaram as composições menos sensíveis às alterações químicas e granulométricas introduzidas pela microssílica. Isto diminui a 
necessidade de reformulação quando se alteram as matériasprimas que compõe o produto.

Considerando a associação da fluidez livre com a análise da etapa de mistura e do comportamento reológico, fica claro que os novos aditivos FS 10 e FS 20 apresentam desempenho superior ao do AC, contrariando a avaliação da fluência livre.

b) Etapa 2: Avaliação do Endurecimento do Concreto (trabalhabilidade)

A Fig. 5 apresenta o comportamento de fluidez em função do tempo, avaliado estática e dinamicamente, para os sistemas AluCAC e Alu-CAC-MS dispersados com os aditivos testados, exceto o DV, que apresentou fluidez inicial muito reduzida ( $\mathrm{FL}<10 \%$ ).

A comparação entre as duas técnicas de medida da variação da fluidez possui importância prática, pois determina se a manutenção do cisalhamento entre a mistura e a aplicação do material é benéfica ou prejudicial à moldagem.

No sistema Alu-CAC, o tempo de trabalhabilidade ( $t_{\text {trab}}$, tempo para que a fluidez seja inferior a $30 \%$ ) estática foi superior àquele obtido de maneira dinâmica, indicando que a manutenção do cisalhamento acelera a pega do concreto. De uma maneira geral, a fluidez das composições diminuiu com o tempo. O AC resultou, para ambos procedimentos de ensaio, os menores valores de $t_{\text {trab }}$, enquanto o FS 20 apresentou o comportamento oposto, sendo o único aditivo que retardou de maneira pronunciada a pega no ensaio estático.

A adição de microssílica alterou o perfil de endurecimento dos concretos. Com exceção do FS 10 (ensaio estático), as demais composições apresentaram um aumento na fluidez, antes de iniciarem o processo de pega. Provavelmente, isto decorre da redução inicial do $\mathrm{pH}$ induzida pela microssílica (Fig. 6), afastando os materiais de sua condição ótima de dispersão. O subsequente aumento de $\mathrm{pH}$ resultou na melhoria da fluidez.

Considerando o tempo de trabalhabilidade, o FS 10 produziu o menor valor, enquanto o FS 20 novamente apresentou a maior capacidade para retardar a pega. Comparando as duas técnicas de medida, os valores de $t_{\text {trab }}$ estática foram moderadamente superiores à dinâmica, excetuando-se o FS 10 que no ensaio estático obteve fluidez inferior a $30 \%\left(t_{\text {trab }}=0\right)$. Sistemas com este aditivo necessitam a manutenção do cisalhamento entre a mistura e a aplicação.

\section{CONCLUSÕES}

Os resultados e as discussões apresentadas neste trabalho confirmam a profunda influência que os aditivos dispersantes exercem sobre o comportamento reológico dos concretos refratários de alta alumina, contendo ou não microssílica.

Considerando-se apenas a técnica de caracterização reológica baseada no ensaio de fluidez livre, o ácido cítrico obteve desempenho igual ou superior aos novos aditivos com moléculas com formato tipo pente, FS 10 e FS 20.

As vantagens desta arquitetura molecular somente foram identificadas pelo método de ensaio proposto neste trabalho (associação da fluidez com a técnica de reometria de concretos), que avalia as etapas de mistura, transporte, moldagem e endurecimento dos concretos.

$\mathrm{O}$ efeito lubrificante das moléculas do tipo pente, que foi mais pronunciado no FS 10, reduziu a energia de mistura e facilitou o fluxo dos materiais em elevadas taxas de cisalhamento, além de resultarem na auto-escoabilidade dos sistemas para que são indicados (FS 10: Alu-CAC; FS 20: AluCAC-MS). Sendo assim, são promissores candidatos para aplicações por fluência livre, bombeamento e projeção.

Os novos dispersantes reduziram também a sensibilidade reológica das composições em relação às alterações químicas e granulométricas introduzidas pela microssílica, indicando uma menor necessidade de reformulação em sistemas comerciais.

Finalmente, o novo método para caracterização reológica é uma necessidade real, tanto na avaliação da influência dos dispersantes, como de qualquer outro fator que influencie a reologia dos concretos refratários.

\section{AGRADECIMENTOS}

Os autores agradecem à FAPESP e a Alcoa Alumínio S.A. pelo apoio dado à execução deste trabalho.

\section{REFERÊNCIAS}

[1] R. G. Pileggi, “Ferramentas para o estudo e desenvolvimento de concretos refratários", Tese de Doutorado, UFSCar, S. Carlos, SP (2001).

[2] A. R. Studart, W. Zhong, V. C. Pandolfelli, "Reological design of zero-cement self-flow high-alumina refractory castables", Am. Ceram. Soc. Bull. 78, 5 (1999) 65-72.

[3] R. G. Pileggi, A. E. Paiva, J. Gallo, V. C. Pandolfelli, "Novel rheometer for refractory castables", Am. Ceram. Soc. Bull. 79, 1 (2000) 54-58.

[4] K. Wutz, "Advanced polymers for monolithic refractories", in Proceedings of Unitecr'01 (2001) 892-903.

[5] R. G. Pileggi, A. R. Studart, V. C. Pandolfelli, "How mixing affects the rheology of refractory castables, part 1", Am. Ceram. Soc. Bull. 80, 6(2001) 27-31.

[6] A. R. Studart, J. Gallo, V. C. Pandolfelli, "Dispersants for highalumina refractory castables", Am. Ceram. Soc. Bull. 81, 4 (2002). [7] I. R. Oliveira, A. R. Studart, R. G. Pileggi, Dispersão e empacotamento de partículas: princípios básicos e aplicações em processamento cerâmico, Fazendo Arte Editorial, São Paulo, (2000) 224.

[8] Y. Tanaka, S. Matsuo, A. Ohta, M. Ueda, “A new admixture for high performance concrete”, Rad. Conc. Tech. (1996) 291-300.

[9] H. Uchikawa, S. Hanehara, D. Sawaki, "The role of steric repulsive force in the dispersion of cement particles in fresh paste prepared with organic admixture", Cem. and Conc. Res., 27, 1 (1997) 37-50.

[10] H. Uchikawa, S. Hanehara, D. Sawaki, A reply to the discussion by Susanta Chaterji of the paper "The role of steric repulsive force in the dispersion of cement particles in fresh paste prepared with organic admixture". Cem. and Conc. Res., 27, 9 (1997) 1453-1455.

[11] P. White, N. C. Fletcher, T. D. Reeves, "Use of fume silica and other ultrafine particles in low cement castables", Proceedings of Unitecr'91, (1991) 259-263.

(Rec. 27/03/02, Ac. 21/09/02) 